\title{
Dissecting 30 Doradus: Optical and Near Infrared Star Formation History of the starburst cluster NGC2070 from the Hubble Tarantula Treasury Project
}

\author{
Michele Cignoni ${ }^{1}$ and the HTTP team \\ ${ }^{1}$ Space Telescope Science Institute, 3700 San Martin Drive, Baltimore, MD, 21218, USA \\ email: cignoni@stsci.edu
}

\begin{abstract}
I will present new results on the star formation history of 30 Doradus in the Large Magellanic Cloud based on the panchromatic imaging survey Hubble Tarantula Treasury Project (HTTP). Here the focus is on the starburst cluster NGC2070. The star formation history is derived by comparing the deepest ever optical and NIR color-magnitude diagrams (CMDs) with state-of-the-art synthetic CMDs generated with the latest PARSEC models, which include all stellar phases from pre-main sequence to post-main sequence. For the first time in this region we are able to measure the star formation using intermediate and low mass stars simultaneously. Our results suggest that NGC2070 experienced a prolonged activity. I will discuss the detailed star formation history, initial mass function and reddening distribution.
\end{abstract}

Keywords. stellar evolution - star forming region: individual, 30 Doradus, galaxies: stellar content

\section{Introduction}

Located in the Large Magellanic Cloud (LMC), 30 Doradus is the most active region in the Local Group. The central area of the nebula is dominated by the starburst region NGC 2070, a collective of several dense sub-clusters (Walborn \& Blades 1997, Sabbi et al. 2012) whose light is dominated by massive OB stars. The most prominent and central of these sub-clusters is the bound super star cluster R 136.

The proximity of the LMC allows to study 30 Doradus with the Hubble Space Telescope (HST) on scales down to $\sim 0.01 \mathrm{pc}$, resolving stars down to the sub-solar regime. However, most of high resolution studies are limited to a few bands and cover only small patches of the 30 Doradus complex. The Hubble Tarantula Treasury Project (HTTP; Sabbi et al. 2013, Sabbi et al. 2015, submitted), a multi-filter HST survey covering a $14 \operatorname{arcmin} \times 12$ arcmin wide area around 30 Doradus at high resolution, fills this gap.

In this work we explore the star formation (SF) during the last $50 \mathrm{Myr}$ for the central 40 pc of 30 Doradus, a.k.a. the starburst region NGC 2070, by comparing the HTTP colormagnitude diagrams (CMDs) with synthetic CMDs. These simulations incorporate the latest (V.1.2S) set of PAdova and TRieste Stellar Evolution Code (PARSEC) isochrones (see Bressan et al. 2012 and Tang et al. 2014), the first theoretical library to include homogeneously all stellar phases from pre-main sequence (PMS) to post-main sequence for all masses between 0.1 and $350 \mathrm{M}_{\odot}$. For the first time, we derive the history of the region using low and intermediate mass stars. In particular, we exploit magnitudes and colors of the PMS Turn-Ons (see e.g. Mayne 2010, Cignoni et al. 2010), i.e. the CMD loci where the PMS phase joins to the MS. 


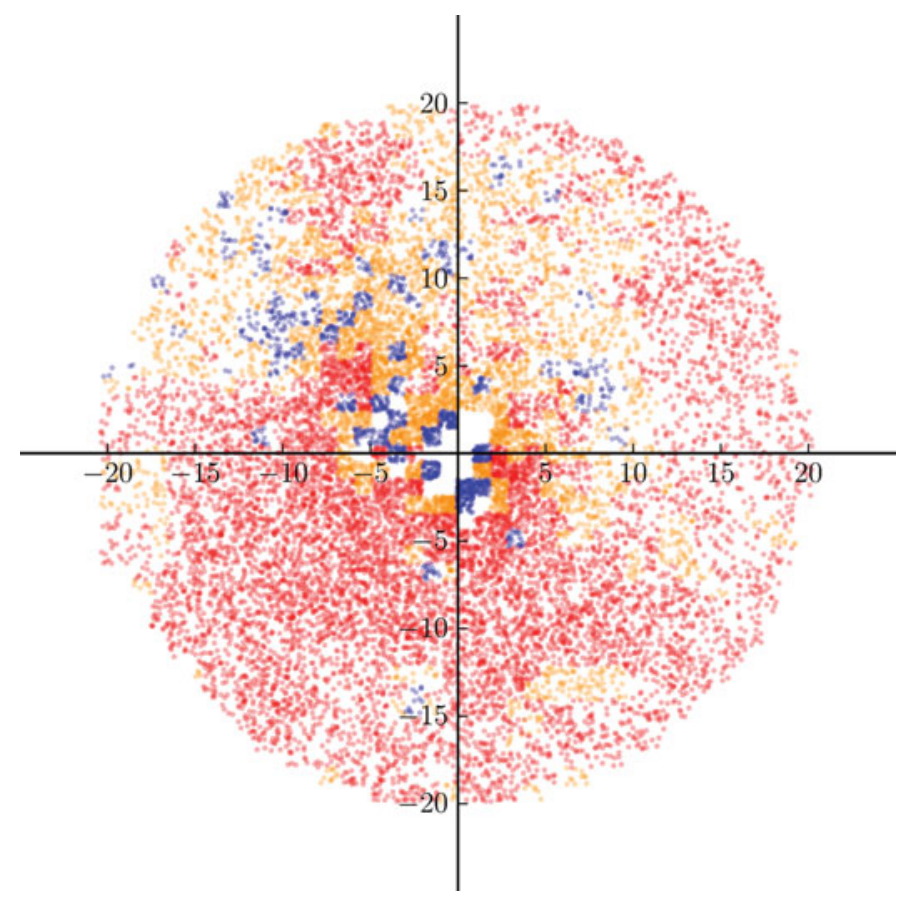

Figure 1. Sub-regions of NGC 2070 where the average completeness is $50 \%$ for $\mathrm{F} 555 \mathrm{~W}>24$ (A1; red dots), $23<\mathrm{F} 555 \mathrm{~W}<24$ (A2; orange dots) and $22<\mathrm{F} 555 \mathrm{~W}<23$ (A3; blue dots).

We use both the optical F555W vs F555W-F775W and the near infrared (NIR) F110W vs F110W-F160W CMDs of the HTTP sample, which offer complementary advantages in terms of higher spatial resolution (the optical) and lower reddening sensitivity (the NIR).

\section{Biases}

In trying to recover the SFH of NGC 2070 we faced three major problems: 1) membership errors due to field interlopers from the LMC field that mimic older populations; 2 ) extreme crowding conditions that exacerbate the incompleteness, shortening the reachable look-back time; 3) high level of differential reddening that spreads and dims the CMD, blending together young PMS stars and older MS stars, which introduces further age ambiguities. Here in the following there are our solutions.

Field contamination: A typical approach is to decontaminate the cluster by subtracting the star-counts from a reference field. However, given reddening and incompleteness variations across the whole 30 Doradus, it is impossible to find another direction replicating the observational conditions of NGC 2070. Our solution was to use a field (within the HTTP field of view; see Section 5.1 in Cignoni et al. 2015 for details), resembling as much as possible the LMC field we would observe without NGC 2070, which means low or negligible SF activity in the last $50 \mathrm{Myr}$, minimal differential reddening and high completeness down to F555W $\sim 24$. This reference LMC field was then artificially corrected for the more severe incompleteness and photometric errors of NGC 2070. The resulting field differs only in terms of normalization and differential reddening from the real LMC field contaminating NGC 2070. Finally, reddening and normalization of this field were tuned together with the SFH (see the next Section) of NGC 2070 until an adequate match of NGC 2070's CMD was found; 


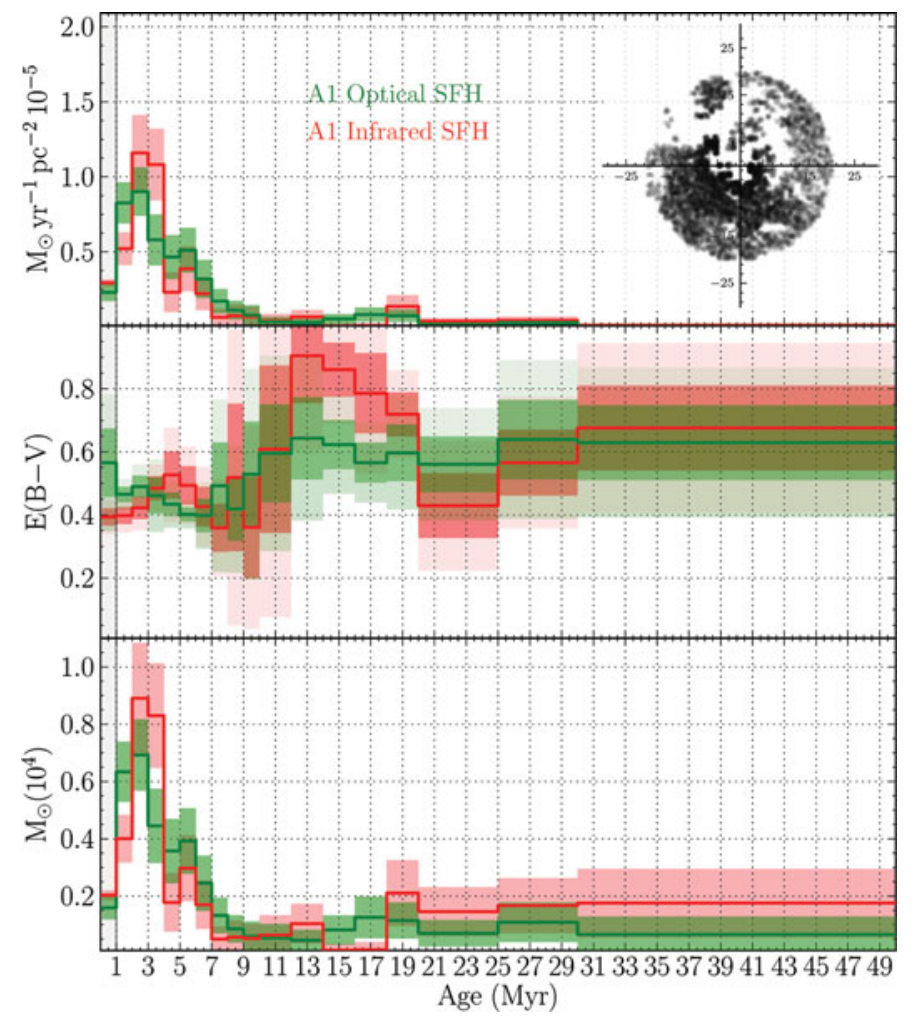

Figure 2. Recovered SFH (top panel), reddening distribution (middle panel) and mass distribution (bottom panel) for the sub-region A1. The optical and NIR solution are plotted in green and red colors, respectively. The bin showing the SF rate in the 0 to $1 \mathrm{Myr}$ range is marked in light grey to indicate that systematic uncertainties may be comparable to or are greater than the formal errors (see text). The inset panel shows the distribution of stars in sub-region A1.

Incompleteness: To test the level of incompleteness of our photometric data and have reliable estimates of photometric errors, we ran extensive artificial star experiments. The experiments consist of adding "fake" sources ( 5 million) for each of the eight pass-bands, modeled with the point spread function used in the photometric analysis of the frames, onto the actual images. We then applied the same source detection routines used for our science images to the fields containing the combined actual images and the fake sources. The results of the test (difference between input and output fake magnitudes, incompleteness) were then convolved with the stellar models;

Differential reddening: The area is strongly affected by differential reddening. Moreover, we do not know where populations of different ages are located inside NGC 2070. For example, young massive stars may have carved the gas with their ionizing flux, hence lowering the extinction. On the other hand, high levels of SF could be sustained only where the gas density is higher, which would lead to the opposite situation. To take into account all these effect, reddening is a free parameter and can vary as a function of age.

\section{The SFH procedure}

In our procedure, the most likely $\mathrm{SFH}$ is the one whose synthetic CMD minimizes the residuals from the observational counterpart. The first step is to create an extensive 


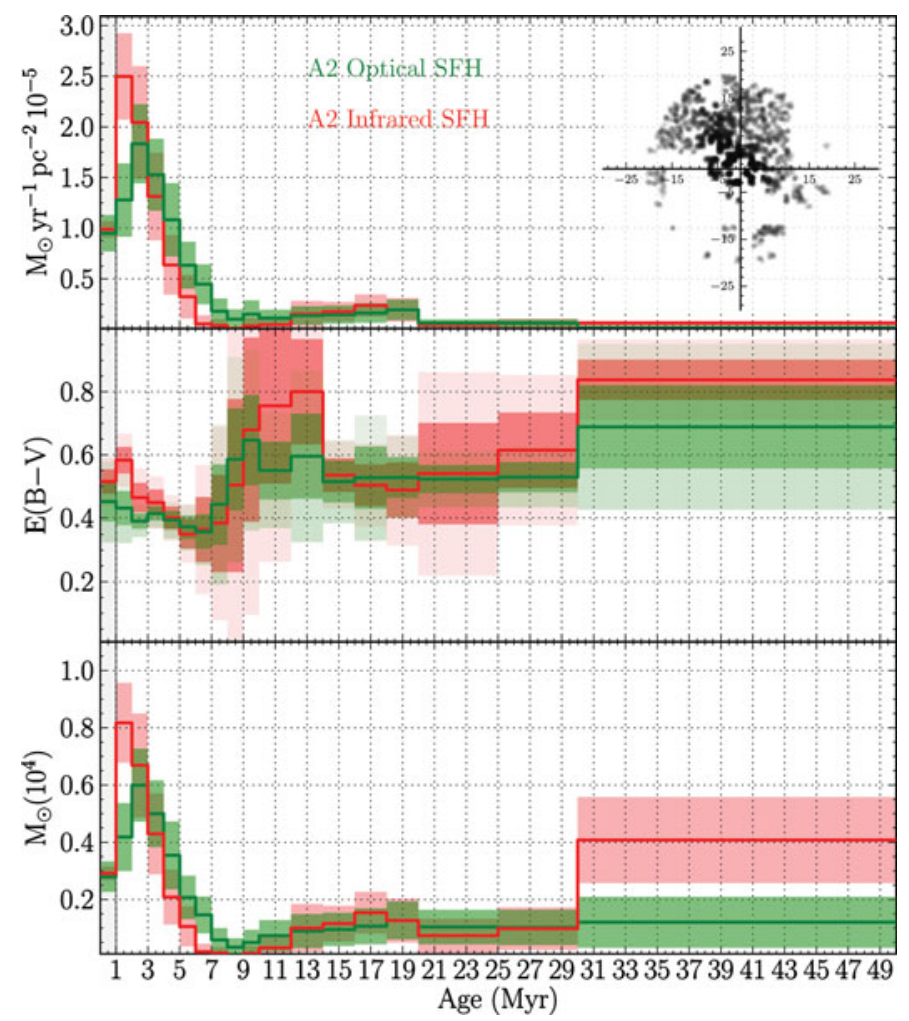

Figure 3. Same as Fig. 2 but for the sub-region A2.

library of basic synthetic CMDs generated from stellar models. Each basic $\mathrm{CMD}_{j, k}$ is a "fuzzy" isochrone, with duration $\Delta t$ and fixed reddening $\mathrm{E}(\mathrm{B}-\mathrm{V})$, populated through a classical Monte Carlo procedure: 1) synthetic masses and ages are extracted from a Kroupa (2001) initial mass function (IMF) and the j-th SF step, respectively; and 2) synthetic masses and ages are converted to absolute synthetic magnitudes and colors by using a fine grid of isochrones. For our calculations we used the latest PARSEC isochrones for the metallicity $\mathrm{Z}=0.008 ; 3)$ a $30 \%$ of synthetic stars is randomly chosen to have a companion. The masses of companions are extracted from the same IMF and their flux is added to the flux of the primaries; 4) The absolute synthetic photometry is put at the distance of the $\mathrm{LMC}\left((\mathrm{m}-\mathrm{M})_{0}=18.5\right.$; see e.g. Panagia et al. 1991) and reddened with the $\mathrm{k}$-th reddening $\mathrm{E}(\mathrm{B}-\mathrm{V})$. To produce realistic simulations, all basic CMDs are degraded with photometric errors and incompleteness as estimated from artificial star tests.

As done previously (Cignoni et al. 2006, Cignoni et al. 2011), we parameterize a generic CMD as a linear superposition of the basic CMDs. The explored ages range from now up to $50 \mathrm{Myr}$ ago. Since older isochrones tend to be more tightly packed in the CMD than younger isochrones, the duration of each age step increases with age from 1 Myr (at the present time) to $20 \mathrm{Myr}$ (50 Myr ago). The reddening is allowed to vary between 0 and $1 \mathrm{mag}$ with a step of $0.05 \mathrm{mag}$, while the extinction coefficients $R_{\lambda}$ are taken from De Marchi \& Panagia (2014). In total we deal with 380 parameters, divided into 18 steps in age (plus one parameter controlling field contamination) and 20 steps in reddening. To explore this wide parameter space we have combined a well tested genetic algorithm 


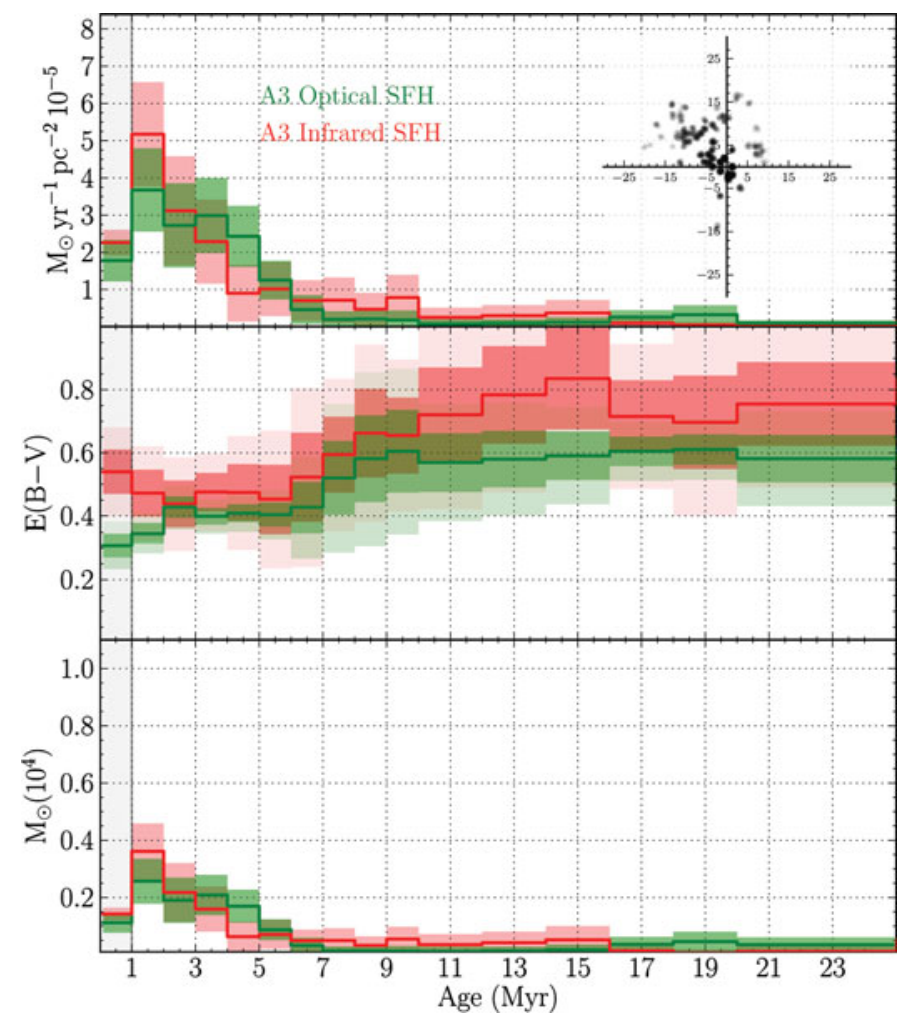

Figure 4. Same as Fig. 2 but for sub-region A3.

(GA), Pikaia†, with a local search routine (see Cignoni et al. 2015 for details). As shown in various papers (see, e.g., $\mathrm{Ng}$ et al. 2002, Aparicio \& Hidalgo 2009, Small et al. 2013), GAs allow to find global optimum more efficiently than a local search alone. In our approach the synergy of the GA and a local search combines the advantages of both worlds.

Which part of the CMD is used? Not all of the CMD is used to recover the SFH. Our analysis is limited on the bright end by the saturation magnitude and on the faint end by the $50 \%$ completeness magnitude level. Nonetheless, even with these conservative selections, the large variations of completeness with magnitude in some regions force us to derive the $\mathrm{SFH}$ in sub-regions within which the completeness is reasonably uniform. Fig. 1 shows our selection: sub-regions of NGC 2070 where the completeness in F555W is $50 \%$ for $\mathrm{F} 555 \mathrm{~W}>24(\mathrm{~A} 1), 23<\mathrm{F} 555 \mathrm{~W}<24(\mathrm{~A} 2)$ and $22<\mathrm{F} 555 \mathrm{~W}<23$ (A3) are indicated in red, orange and blue, respectively. The stars found in both the F555W and F775W filters inside these sub-regions are used to recover the optical SFHs. Overall, our sub-regions trace the stellar density, from the lowest (A1) to the highest (A3).

This analysis leaves out the very center of NGC 2070, $\mathrm{a} \approx 3$ pc region mostly represented by $\mathrm{R} 136$.

$\dagger$ Routine developed at the High Altitude Observatory, and available in the public domain http://www.hao.ucar.edu/modeling/pikaia.php. 


\section{Best SFH for NGC 2070}

Figures 2 and 3 show the recovered $\mathrm{SF}$ rate (in $\mathrm{M}_{\odot} \mathrm{yr}^{-1} \mathrm{pc}^{-2}$; top panel), reddening (middle panel) and stellar mass (bottom panel) as a function of time for sub-regions A1 and A2 respectively, as predicted using the optical (green curve) and NIR (red curve) CMDs. Uncertainties are the quadrature sum of a statistical error (obtained by bootstrapping the data and re-deriving the solutions) and systematic error (obtained by re-deriving the solutions with different age-binnings and CMD binning scheme).

Despite the different spatial resolution and reddening sensitivity, optical and NIR predictions are in good agreement. Both results predict that mild SF activity started throughout the whole NGC 2070 region $\approx 20$ Myr ago, and that about 7-8 Myr ago the birth rate accelerated.

Focusing on the specific sub-regions, the SFH of sub-region A1 shows a mild bimodality, with a minor peak around 5-7 Myr ago and a major peak 1-4 Myr ago (1-3 Myr ago in the optical SFH, 2-4 Myr ago in the NIR one). The measured reddening distribution anti-correlates with $\mathrm{SF}$ : the average $\mathrm{E}(\mathrm{B}-\mathrm{V})$ is $\approx 0.6$ for stars with ages older than 7 $\mathrm{Myr}$, when the SF activity was lower, and $\approx 0.4$ for stars of younger ages, when the $\mathrm{SF}$ was stronger. Optical and NIR reddening derivations agree within errors, both in confirming the presence of notable differential reddening.

The SF in sub-region A2 (Fig. 3) is about two times higher than in sub-region A1. The most important feature in the $\mathrm{A} 2 \mathrm{SF}$ is a pronounced peak in both optical and NIR solutions 1-3 Myr ago. Like in sub-region A1, the reddening of sub-region A2 is anti-correlated with the SF activity.

Fig. 4 shows the SFH in the sub-region A3 over the last $25 \mathrm{Myr}$ (the crowding conditions prevented to investigate earlier activity). The peak activity in both the optical and NIR solution is more than two times higher than in A2, and at odds with other sub-regions, is clearly located between 1 and 2 Myr ago (more pronounced in the NIR solution).

\section{Conclusions}

We summarize here our main conclusions:

SFH: NGC 2070 experienced prolonged activity. We identify three major events in the history of this star forming region: 1$) \approx 20 \mathrm{Myr}$ ago - This epoch demarcates the commencement of the first significant period of SF; 2) 7 Myr ago the SF accelerated throughout the entire region; 3) 1-3 Myr ago the activity reached a peak. In this time range, the $\mathrm{SF}$ moves from the periphery to the central regions. Our innermost sub-region (A3) shows a maximum activity 1-2 Myr ago;

Reddening: we find an average $\mathrm{E}(\mathrm{B}-\mathrm{V}) \approx 0.4 \mathrm{mag}$ for the young population $(<10 \mathrm{Myr}$ old $)$, and $\approx 0.6 \mathrm{mag}$ for the old one ( $>10$ Myr old);

$I M F$ : Except the innermost few pc, where the incompleteness is too severe to allow firm conclusions, a Kroupa IMF in the range $0.5-7 \mathrm{M}_{\odot}$ is compatible with the data.

\section{References}

Aparicio, A. \& Hidalgo, S. L. 2009, AJ, 138, 558

Bressan, A., Marigo, P., Girardi, L., et al. 2012, MNRAS, 427, 127

Cignoni, M., Degl'Innocenti, S., Prada Moroni, P., \& Shore, S. N. 2006, A\&AA, 459, 783

Cignoni, M., Tosi, M., Sabbi, E., et al. 2010, ApJL, 712, L63

Cignoni, M., Tosi, M., Sabbi, E., Nota, A., \& Gallagher, J. S. 2011, AJ, 141, 31

Cignoni, M., Sabbi, E., van der Marel, R. P., et al. 2015, arXiv:1505.04799

De Marchi, G. \& Panagia, N. 2014, MNRAS, 445, 93 
Hunter, D. A., Shaya, E. J., Holtzman, J. A., et al. 1995, ApJ, 448, 179

Kroupa, P. 2001, MNRAS, 322, 231

Mayne, N. J. 2010, MNRAS, 408, 1409

Ng, Y. K., Brogt, E., Chiosi, C., \& Bertelli, G. 2002, A\& A, 392, 1129

Panagia, N., Gilmozzi, R., Macchetto, F., Adorf, H.-M., \& Kirshner, R. P. 1991, ApJL, 380, L23

Sabbi, E., Lennon, D. J., Gieles, M., et al. 2012, ApJL, 754, LL37

Sabbi, E., Anderson, J., Lennon, D. J., et al. 2013, AJ, 146, 53

Small, E. E., Bersier, D., \& Salaris, M. 2013, MNRAS, 428, 763

Tang, J., Bressan, A., Rosenfield, P., et al. 2014, MNRAS, 445, 4287

Walborn, N. R. \& Blades, J. C. 1997, ApJS, 112, 457 